\title{
The transcription factor FoxM1 activates Nurr1 to promote intestinal regeneration after ischemia/ reperfusion injury
}

\author{
Guo Zu', Jing Guo ${ }^{2}$, Tingting Zhou ${ }^{3}$, Ningwei Che ${ }^{4}$, Baiying Liu', Dong Wang ${ }^{1}$ and Xiangwen Zhang ${ }^{1}$
}

\begin{abstract}
FoxM1 is involved in the regeneration of several organs after injury and expressed in the intestinal mucosa. The intrinsic mechanism of FoxM1 activity in the mucosa after intestinal ischemia/reperfusion (I/R) injury has not been reported. Therefore, we investigated the role of FoxM1 in mediating intestinal mucosa regeneration after I/R injury. Expression of FoxM1 and the proliferation of intestinal mucosa epithelial cells were examined in rats with intestinal I/R injury and an IEC-6 cell hypoxia/reperfusion (H/R) model. The effects of FoxM1 inhibition or activation on intestinal epithelial cell proliferation were measured. FoxM1 expression was consistent with the proliferation of intestinal epithelial cells in the intestinal mucosa after I/R injury. Inhibition of FoxM1 expression led to the downregulation of Ki67 expression mediated by the inhibited expression of Nurr1, and FoxM1 overexpression promoted IEC-6 cell proliferation after $\mathrm{H} / \mathrm{R}$ injury through activating Nurr1 expression. Furthermore, FoxM1 directly promoted the transcription of Nurr1 by directly binding the promoter of Nurr1. Further investigation showed low expression levels of FoxM1, Nurr1, and Ki-67 in the intestinal epithelium of patients with intestinal ischemic injury. FoxM1 acts as a critical regulator of intestinal regeneration after I/R injury by directly promoting the transcription of Nurr1. The FoxM1/ Nurr1 signaling pathway represents a promising therapeutic target for intestinal I/R injury and related clinical diseases.
\end{abstract}

\section{Introduction}

Intestinal ischemia/reperfusion (I/R) injury is a common pathophysiological process in many clinical settings that includes small bowel transplantation, hemorrhagic shock, and necrotizing enterocolitis ${ }^{1,2}$. It can cause severe intestinal mucosa damage that provokes intestinal mucosal barrier dysfunction. Once the intestinal epithelium, one of the most rapidly proliferating tissues in the body, is damaged, it activates regeneration programs to restore its mucosal barrier function ${ }^{3}$. The intrinsic mechanism of intestinal mucosa regeneration is not always sufficient to restore mucosal barrier function damaged by $I / R$ injury, which is associated with significant

\footnotetext{
Correspondence: Guo Zu (zushanghai@163.com)

${ }^{1}$ Department of Gastroenterology Surgery, The Dalian Municipal Central Hospital Affiliated of Dalian Medical University, 116033 Dalian, China ${ }^{2}$ Institute of Integrative Medicine, Dalian Medical University, 116044 Dalian, China

Full list of author information is available at the end of the article.

This author contributed equally: Jing Guo
}

morbidity and mortality. The pathophysiology of intestinal regeneration after $\mathrm{I} / \mathrm{R}$ injury is complex and involves many signaling pathways ${ }^{4-6}$. Several signaling pathways are involved in the proliferation of intestinal epithelial cells after $\mathrm{I} / \mathrm{R}$ injury ${ }^{7}$. However, the intrinsic mechanisms of intestinal epithelial cell proliferation after $\mathrm{I} / \mathrm{R}$ injury are still not known.

As a typical transcription factor, FoxM1 belongs to the family of Forkhead box (Fox) proteins and is associated with cell proliferation. It is expressed in several embryonic tissues and the testes, thymus and intestinal crypts in adult mice ${ }^{8-10}$. In addition, FoxM1 is a key regulator of cell cycle progression and critical for the replication of DNA and mitosis ${ }^{11-13}$. Studies have shown that FoxM1 expression is reactivated after organ injury and that FoxM1 has pleiotropic roles during mouse liver regeneration after partial hepatectomy injury ${ }^{14}$. Ackermann reported that FoxM1 is required for the proliferation of preexisting beta cells after $60 \%$ partial pancreatectomy ${ }^{15}$. 
Ye et al. demonstrated that the expression of FoxM1 accelerates DNA replication and hepatocyte mitosis in the regenerating liver ${ }^{16}$. FoxM1, a key regulator of quiescence and self-renewal in hematopoietic stem cells, is mediated by control of Nurr1 expression ${ }^{17}$, and our previous research found that Nurr1 promotes intestinal mucosa epithelial cell proliferation after $\mathrm{I} / \mathrm{R}$ injury by inhibiting p21 expression ${ }^{18}$. FoxM1, which is collectively considered a typical proliferation-associated transcription factor, is expressed in intestinal crypts. However, the effects of FoxM1 in regeneration of the intestinal mucosa after intestinal injury have not been examined.

Here, we propose that FoxM1 plays an important role in promoting intestinal mucosa regeneration after I/R injury. We determined that FoxM1 promotes intestinal mucosa epithelial cell proliferation via promoting the expression of Nurr1. Mechanistically, our findings demonstrate the direct transcriptional regulation of Nurr1 by FoxM1 in intestinal mucosa regeneration after $I / R$ injury and that the FoxM1/Nurr1 pathway is involved in intestinal regeneration after $I / R$ injury, providing new and potential therapies for intestinal I/R injury.

\section{Materials and methods}

\section{Intestinal $\mathrm{I} / \mathrm{R}$ injury model and tissue analysis}

Male wild-type Sprague-Dawley rats weighing between 180 and $220 \mathrm{~g}$ were purchased from the Animal Center of Dalian Medical University. The animal studies were performed at Dalian Medical University. The intestinal I/R injury model was described in a previous study in rats ${ }^{19}$. Briefly, after anesthetization of the rats with an intraperitoneal injection of pentobarbital $(40 \mathrm{mg} / \mathrm{kg})$, the superior mesenteric artery (SMA) and collateral vessels were interrupted with atraumatic clips. After $1 \mathrm{~h}$ of ischemia, the atraumatic clips were removed to initiate reperfusion for $3,6,12$, or $24 \mathrm{~h}$. Ileum tissue samples $(1 \mathrm{~cm})$ were collected for the various experimental evaluations required for this study. Rats in the sham group underwent laparotomy without SMA and collateral vessel occlusion. Rats in the sham group did not exhibit changes in FoxM1 expression, and pentobarbital anesthesia did not influence FoxM1 expression (supplementary material 1).

To test the roles of FoxM1 in intestinal mucosa regeneration after I/R injury, we used the FoxM1 inhibitor thiostrepton (TST) to inhibit the expression of FoxM1 $1^{20,21}$. Rats were randomly divided into 4 groups: the sham, sham+TST, I/R, and I/R+TST groups. The sham and I/R groups were treated as described above, and $50 \mathrm{mg} / \mathrm{kg}$ TST was given daily by intraperitoneal injection for two days before I/R surgery. After $1 \mathrm{~h}$ of intestinal ischemia followed by $6 \mathrm{~h}$ of reperfusion, ileum tissue samples were collected.

To determine whether Nurr1-mediated FoxM1 promotes intestinal regeneration after $I / R$ injury, we determined whether the Nurr1 activator 1,1-bis(39-indolyl)-1-(p-chlorophenyl) methane (C-DIM12) ${ }^{22,23}$ could abolish the inhibitory effects of TST on intestinal I/R injury. Rats were randomly divided into 4 groups: the sham, I/R, I/R+TST, and I/R+TST+C-DIM12 groups. Rats in the sham, I/R and I/R+TST groups were treated as described above. C-DIM12 $(50 \mathrm{mg} / \mathrm{kg})$ was given before surgery. After $1 \mathrm{~h}$ of intestinal ischemia followed by $6 \mathrm{~h}$ of reperfusion, ileum tissue samples were collected.

The protocols and experiments in this study were conducted according to the guidelines of Dalian Municipal Central Hospital Affiliated of Dalian Medical University and approved by the Institutional Ethics Committee of Dalian Municipal Central Hospital Affiliated of Dalian Medical University.

\section{Western blot analysis}

Protein expression in the intestinal samples or IEC-6 cells was analyzed by western blotting. The samples or cells were washed with ice-cold PBS and lysed in RIPA buffer (P0013, Beyotime, China) supplemented with phosphatase inhibitors and a protease inhibitor (P1048, Beyotime, China). Cells lysates were incubated for $2 \mathrm{~h}$ at $4{ }^{\circ} \mathrm{C}$ and centrifuged at $13,000 \mathrm{rpm}$ for $10 \mathrm{~min}$ at $4{ }^{\circ} \mathrm{C}$ to remove cellular debris. The protein concentration was determined with a BCA protein assay kit (P0010, Beyotime, China). Whole cell lysates were loaded on a $12 \%$ SDS-polyacrylamide gel and transblotted to a nitrocellulose membrane after electrophoretic separation. Blocking was carried out in 5\% PBS-milk, following which the membrane was incubated with anti-FoxM1 antibody (1:500, Santa Cruz Biotechnology, USA) and anti-Nurr1 antibody (1:500, Cell Signaling Technology, USA) overnight. The membrane was washed with $1 \times$ PBST and probed with HRP-conjugated anti-rabbit antibody (1:1000 dilution) for $2 \mathrm{~h}$ at $37^{\circ} \mathrm{C}$, followed by enhanced ECL chemiluminescence (P0018, Beyotime, China) detection. As a loading control, membranes were incubated with anti- $\beta$-actin antibody (TA-09, ZSGB-BIO, China) for $2 \mathrm{~h}$ at $37^{\circ} \mathrm{C}$, washed and probed with HRP-conjugated antimouse or anti-rabbit antibody. The results were calculated using the ratio of the density of the protein of interest corrected by the intensity of the protein used as the control ( $\beta$-actin).

\section{Real-time PCR}

Total RNA from intestinal tissues (in vivo) and IEC-6 cells (in vitro) was extracted. First-strand cDNA was synthesized, and target cDNA was amplified. The PCR primers were as follows: rat FoxM1 F: 5'-CAAG GTAAAAGCCACGTCTAAG C-3', R: 5'-GGAGCAGC AGGTGACTAATGG-3'; rat Nurr1 F: 5'-CCAA TCCGGC AATGACCAG-3', R: 5'-TGATGATCTCC ATAGAGCCAGTCAG-3'; rat $\beta$-actin F: $5^{\prime}$-CTGG 
AGAAGAGCTATGAGCTG-3'， R: 5'-AATCTCC TTC TGAT CCTGTC-3'; human FoxM1 F: 5'-GGAGGA AATGCCACACTTAGCG-3'， R: 5'-TAGGACTTCTT GGGTCTTGGGGTG-3'; human Nurr1 F: 5'-CATGG ACCT CACCAACACTG-3', R: 5'-AGTAAACCGACC CGGAGTG-3'; and human $\beta$-actin $\mathrm{F}$ : 5'-ACCCTG AAGTACCCCATCGAG-3'; R: 5'-ACATGATCTGGGT CATCTTCTCG-3'. mRNA expression was quantified using a TransStart Top Green qPCR SuperMix kit (TransGen Biotech, Beijing, China). $\beta$-Actin was used as an endogenous control, and the $\Delta \Delta C T$ method was used to analyze relative mRNA expression.

\section{Histological and immunohistochemical staining}

Histological and immunohistochemical staining was performed on paraffin-embedded intestinal tissue. Immunohistochemical staining was performed using primary antibody against Ki-67, a nuclear protein associated with cell proliferation (ab15580, 1:200 dilution, Abcam, USA). Then, a streptavidin-biotin-peroxidase kit (ZSGBBIO, Beijing, China) was used according to the manufacturer's instructions. We measured the proliferation index, which was calculated as the average number of Ki67 -positive enterocytes per 100 enterocytes. At least 2000 enterocytes were assessed for these calculations ${ }^{18}$. Analysis and evaluation of the immunostaining results were independently carried out by two authors. Differences of opinion were reassessed together to reach a consensus.

\section{Cell culture and hypoxia/reoxygenation $(\mathrm{H} / \mathrm{R})$ model}

IEC-6 cells were cultured, and an H/R model was developed as previously described ${ }^{18}$. IEC-6 cells were obtained from American Type Culture Collection (Manassas, VA, USA) and cultured at $37^{\circ} \mathrm{C}$ in a $5 \% \mathrm{CO}_{2}$ incubator. Dulbecco's modified Eagle's medium (DMEM; Gibco BRL) was used as the cell medium. IEC-6 cells were incubated under hypoxic conditions $\left(5 \% \mathrm{CO}_{2}, 1 \% \mathrm{O}_{2}\right.$, and $94 \% \mathrm{~N}_{2}$ ).

\section{siRNA transfection}

Sequence-specific siRNA was used for RNA interference in IEC-6 cells using Lipofectamine 2000 (Invitrogen, Shanghai, China). The siRNA sequences against FoxM1 were as follows: si-FoxM1: sense, $5^{\prime}$-GGAUG UGACUUACAUCGUUtt-3'; antisense, 5'-AACGAUGU AAGUCACAUCCtt- $3^{\prime}$. The effects of each siRNA were analyzed using RT-PCR and western blotting.

\section{Cell proliferation assay}

The proliferation of IEC-6 cells was assessed using a Cell Counting Kit-8 assay (Dojindo Molecular Technologies, Inc., Tokyo, Japan) according to the manufacturer's recommendations. A 96-well plate was seeded with IEC-6 cells at a total density of $2 \times 10^{3}$ cells/well in $100 \mu \mathrm{L}$ of
DMEM. Cells were allowed to attach and then subjected to $H / R$. At the indicated time points, the cells were washed with $100 \mu \mathrm{L}$ of DMEM. The cells were incubated for $2 \mathrm{~h}$ in $10 \mu \mathrm{L}$ of CCK- 8 solution, and the absorbance at $450 \mathrm{~nm}$ was measured using an ELISA microplate reader (Thermo Scientific, USA).

\section{Plasmid construction and transient transfection}

pEX-3 (pGCMV/MCS/Neo) plasmids expressing FoxM1 or Nurr1 were synthesized and purchased from GenePharma (Shanghai, China). The pEX-3 plasmid or control vector $(2 \mu \mathrm{g})$ were used to transfect IEC- 6 cells. After transfection using Lipofectamine 2000 for $48 \mathrm{~h}$, G418 $(400 \mu \mathrm{g} / \mathrm{mL})$ was used for cell selection. Stably transduced IEC-6 cells were used for subsequent experiments.

\section{Chromatin immunoprecipitation (ChIP) assay}

A ChIP assay kit (Beyotime, Shanghai, China) was used for ChIP assays performed as previously described ${ }^{18}$. Briefly, cells were treated with paraformaldehyde. Quenched cells were lysed, followed by sonication. A specific antibody $(2 \mu \mathrm{g})$ was used for immunoprecipitation. DNA/ protein complexes were eluted in elution buffer. Purified DNA was used for PCR analysis. PCR products were generated using the following primers: rat Nurr1 site 1: F, 5'-ATCCCATCTGGCTGACTTGT-3'， R, 5'-TCAGCA AATCTTA GCAACCGT-3'; Nurr1 site 2: F, 5'-GG GATTCCAGGGTGTGCTAT-3'， R, 5'-TCA GCAAA TCTTAGCAACCGT-3'; and rat GAPDH: F, 5'-CGTAG CTCAGGCCTCTG CGCCCTT-3', R, 5'-GCACTGC ACAAGAAGAT GCGGCTG-3'.

\section{Luciferase reporter assay}

The wild-type rat Nurr1 promoter and mutated Nurr1 promoter, that harbored a mutation in the FoxM1binding motif were synthesized by GenePharma (Shanghai, China). The wild-type and mutated promoters were separately cloned into the pGL3-Basic luciferase vector between its NheI and HindIII sites. When IEC-6 cells reached $60-70 \%$ confluence, they were transfected with plasmid $(2 \mu \mathrm{g} /$ well $)$ with Lipofectamine 2000. After transfection for $48 \mathrm{~h}$, the cells were lysed with cell lysis buffer, and dual-luciferase reporter assay reagents (TransGen Biotech, Beijing, China) were then used according to the manufacturer's instruments. Luciferase activities were determined and normalized to Renilla luciferase activity.

\section{Immunofluorescence}

In vivo intestinal tissue samples were cryosectioned (10 $\mu \mathrm{m}$ thickness), and IEC-6 cells were postfixed in $4 \%$ paraformaldehyde in vitro. Then, tissues and cells were incubated with a primary antibody. The primary 
antibodies used were as follows: primary anti-Ki-67 (ab15580, 1:200 dilution, Abcam, USA) and primary anti-phosphorylated histone $\mathrm{H} 3$ (pH3, a mitosis marker) (1:50, Biogot Technology, Co., Ltd., Nanjing, China). Then, a secondary antibody (Invitrogen Life Technologies, Carlsbad, CA, USA) and DAPI (Beyotime, Shanghai, China) were added. A Leica DM 4000B microscope was used to examine staining. We also measured the proliferation index, which were the same as those of immunohistochemical staining, to analyze and evaluate proliferation of the intestinal mucosa.

\section{Patients}

Five ischemic intestinal samples were collected from 5 clinical patients who underwent intestinal ischemia at Dalian Municipal Central Hospital Affiliated of Dalian Medical University. Written informed consent was obtained from the families of patients. A total of 5 patients underwent an operation for acute mesenteric arterial embolism, strangulated intestinal obstruction or incarcerated hernia. We excluded patients with tumors or inflammatory bowel disease, patients who took immunosuppressants and patients who disagreed. The patients consisted of four males and one female with an average age of 66.6 years. The ischemia duration for the intestinal samples was greater than $6 \mathrm{~h}$. After excision of ischemic intestinal tissue, small intestine tissue samples were harvested and stored immediately in liquid nitrogen for western blotting and PCR analysis. Tissues from another part of the intestine were fixed in $10 \%$ formalin for histological and immunohistochemical staining. Intestinal samples were obtained with the approval of the Institutional Ethical Committees of Dalian Municipal Central Hospital Affiliated of Dalian Medical University.

\section{Statistical analysis}

All values are expressed as the means \pm SDs. Analysis of data between two groups was performed using two-tailed Student's $t$-tests. One-way analysis of variance and Student-Newman-Keuls tests were used to compare means among more than two groups. All data analyses were performed with Statistical Product and Service Solutions and GraphPad Prism 5.0. $P<0.05$ indicated significance.

\section{Results}

\section{FoxM1 is induced during intestinal mucosa regeneration} after $\mathbf{I} / \mathbf{R}$

We previously investigated intestinal epithelium regeneration after $1 \mathrm{~h}$ of ischemia and $3,6,12$, or $24 \mathrm{~h}$ of reperfusion ${ }^{18}$. To determine the expression of FoxM1 in intestinal mucosa regeneration after $I / R$ injury, we tested the expression of FoxM1 in intestinal tissues that underwent $1 \mathrm{~h}$ of ischemia followed by $3,6,12$, or $24 \mathrm{~h}$ of reperfusion. The protein and mRNA expression levels of FoxM1 were tested after reperfusion for different lengths of time (Fig. 1a, b). The mRNA and protein expression levels of FoxM1 were consistent with proliferation of the

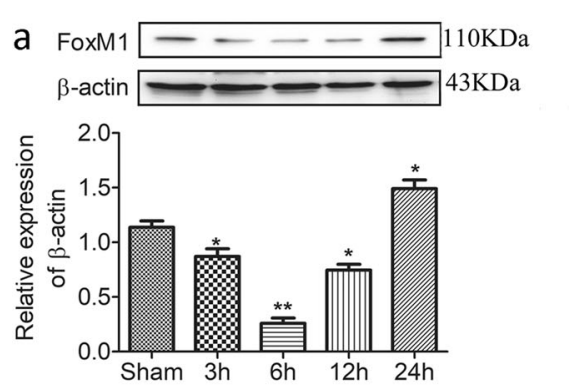

\section{b}
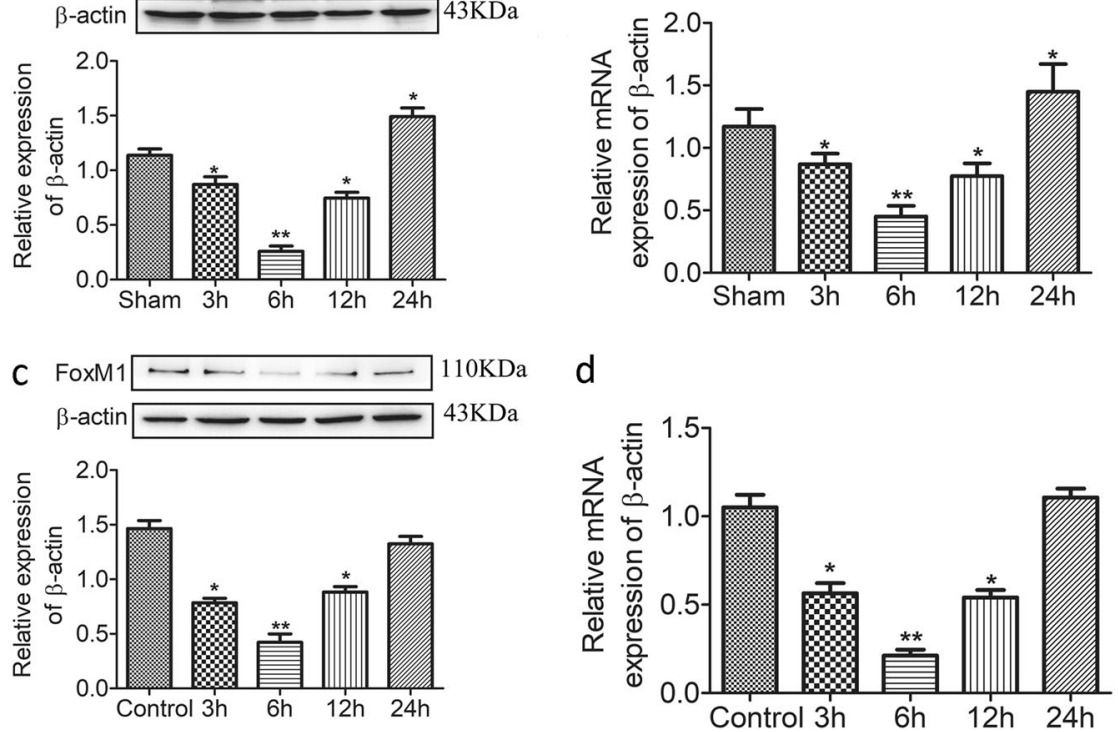

Fig. 1 FoxM1 is induced during intestinal regeneration after $\mathrm{I} / \mathbf{R}$ in vivo and in vitro. $\mathbf{a}$, $\mathbf{b}$ Representative protein and mRNA levels of FoxM1 in rats subjected to ischemia for $1 \mathrm{~h}$ followed by $3,6,12$, or $24 \mathrm{~h}$ of reperfusion $(n=6)$. $\mathbf{c}$, $\mathbf{d}$ Representative protein and mRNA levels of FoxM1 in IEC- 6 cells subjected to $6 \mathrm{~h}$ of hypoxia followed by $3,6,12$, or $24 \mathrm{~h}$ of reoxygenation $(n=6)$. The values are presented as the means \pm SDs. ${ }^{*} P<0.05$ compared to the sham group or control group, ${ }^{* *} P<0.01$ compared to the sham group or control group 
intestinal epithelium after I/R injury. These results indicated that FoxM1 is induced during intestinal mucosa regeneration after I/R injury in vivo.

We also examined FoxM1 expression in IEC-6 cells in vitro. After $6 \mathrm{~h}$ of hypoxia and 3, 6, 12, or $24 \mathrm{~h}$ of reoxygenation, the expression of FoxM1 was associated with IEC-6 cell proliferation after I/R injury (Fig. 1c, d). Overall, these findings suggested that FoxM1 is induced during the proliferation of IEC-6 cells after $H / R$ in vitro.

\section{Reduced expression of FoxM1 inhibits intestinal epithelial proliferation after $\mathrm{I} / \mathrm{R}$ in vivo}

As FoxM1 was induced during mucosa regeneration after intestinal I/R injury, we used the FoxM1 inhibitor TST to test the effect of FoxM1 on intestinal epithelial proliferation $^{20,24}$. First, the data suggested that TST inhibited the expression of FoxM1 (Fig. 2a, b). Then, we investigated the effects of TST on intestinal epithelial proliferation and histology after I/R injury. The I/R group showed impaired intestinal villi and reduced proliferation in the intestinal epithelium compared with the sham group (Fig. 2c, d). The group treated with TST following intestinal $I / R$ injury showed worsened injury and an increased Chiu score compared to the I/R group. In addition, proliferation in the intestinal epithelium after I/R injury was further reduced (Fig. 2e, h). Collectively, these findings suggested that the reduced expression of FoxM1 inhibits intestinal epithelial proliferation induced by intestinal $\mathrm{I} / \mathrm{R}$.

\section{FoxM1 promotes IEC- 6 cell proliferation after H/R injury}

As reduced expression of FoxM1 inhibited intestinal epithelial proliferation after $I / R$, we next tested the effect of FoxM1 knockdown and overexpression on IEC-6 cell proliferation after $\mathrm{H} / \mathrm{R}$ injury. FoxM1 knockdown using siRNA (siFoxM1), FoxM1 overexpression using plasmid transfection (Fig. 3a, b) and IEC-6 cell proliferation after $12 \mathrm{~h}$ and $24 \mathrm{~h}$ of reoxygenation following $6 \mathrm{~h}$ of hypoxia were tested by the CCK- 8 assay and determining the expression of Ki-67. FoxM1 knockdown dramatically decreased the proliferation of IEC- 6 cells compared with that in the H/R group, whereas FoxM1 overexpression increased the proliferation of IEC- 6 cells compared with that in the H/R group (Fig. 3c, d). These results showed that FoxM1 promotes the proliferation of IEC- 6 cells after $\mathrm{H} / \mathrm{R}$ injury.

Nurr1 is induced by FoxM1 during intestinal regeneration after $\mathrm{I} / \mathrm{R}$ injury in vivo and in vitro

As a transcriptional factor, Nurr1 promotes intestinal mucosa regeneration after I/R injury ${ }^{23}$. Hou reported that FoxM1 can regulate Nurr1 expression in mouse and human leukemia cells ${ }^{17}$. Thus, we examined whether FoxM1-mediated intestinal epithelial proliferation is mediated by Nurr1. As expected, Nurr1 and FoxM1 expression levels were similarly inhibited in intestinal tissues after I/R injury (Fig. 4a, b). Similar results were demonstrated following the knockdown and overexpression of FoxM1 in IEC-6 cells after H/R injury (Fig. $4 c, d)$. These results indicated that Nurr1 is induced by FoxM1 during intestinal regeneration after $I / R$ injury in vivo and in vitro.

FoxM1 promotes intestinal regeneration after I/R injury in a Nurr1-dependent manner

As Nurr1 expression is closely associated with FoxM1 activation, we hypothesized that FoxM1 promotes intestinal regeneration after $I / R$ injury in a Nurr1dependent manner. We used the Nurr1 activator CDIM12 to examine the role of Nurr1 in the inhibition of FoxM1. Compared with the I/R group, the group treated with the FoxM1 inhibitor exhibited decreased FoxM1 expression (Fig. 5a, b). Furthermore, the FoxM1 inhibitor obviously inhibited proliferation of the intestinal epithelium after I/R injury. Nurr1 expression in the intestine was activated with the FoxM1 inhibitor TST, and TST no longer exerted any inhibitory effects of proliferation of the intestinal epithelium after I/R injury (Fig. 5c-f). These results showed that FoxM1 promotes intestinal regeneration after $\mathrm{I} / \mathrm{R}$ injury in a Nurr1-dependent manner.

\section{FoxM1 promotes IEC- 6 cell proliferation after $H / R$ injury in a Nurr1-dependent manner}

Our results have showed that FoxM1 promotes intestinal regeneration after $I / R$ injury in a Nurr1-dependent manner in vivo. We further detected the effect of Nurr1 overexpression in proliferation of IEC-6 cells with FoxM1 knockdown after $H / R$ injury. The results showed that Nurr1 was overexpressed in IEC-6 cells with FoxM1 knockdown by plasmid transfection (Fig. 6a, b). Overexpression of Nurr1 reversed the inhibition of IEC- 6 cell proliferation induced by FoxM1 knockdown (Fig. 6c, d). These results suggest that FoxM1 promotes IEC-6 cell proliferation after $H / R$ injury in a Nurr1-dependent manner.

\section{FoxM1 regulates Nurr1 expression by directly inhibiting Nurr1 expression}

Our previous results showed that FoxM1 promotes intestinal mucosa regeneration after I/R injury in a Nurr1dependent manner. In addition, Hou reported that FoxM1 can directly regulate Nurr1 transcription in mice. To test the relationship between FoxM1 and Nurr1 in rats, we searched the proximal region of the Nurr1 promoter for the consensus FoxM1-binding site [T(G/A)TTT(G/A) $\mathrm{TT}]^{25}$. Two putative FoxM1-binding sites were identified upstream of the Nurr1 transcription start site (TSS) (Fig. 7a). In addition, a ChIP assay using IEC-6 cells showed 
a

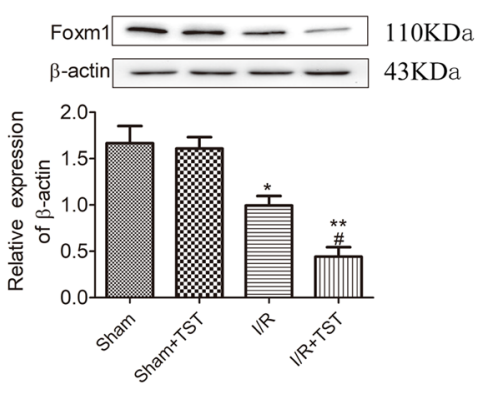

C
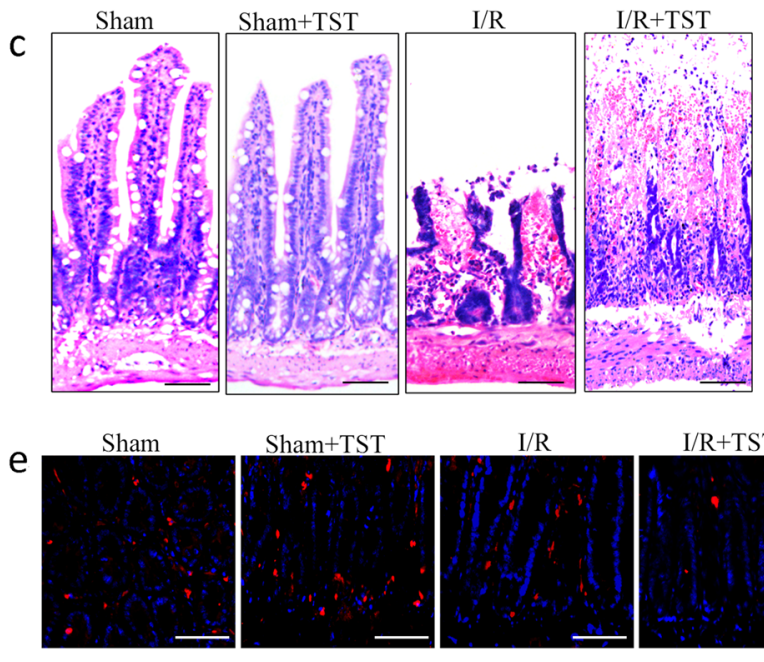

Sham + TST
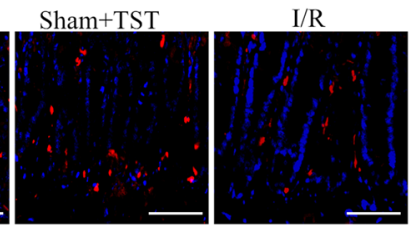

g

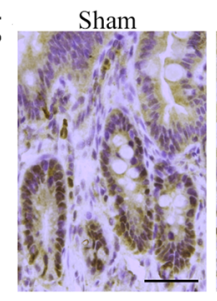

Sham + TST

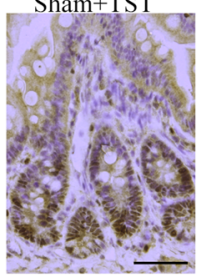

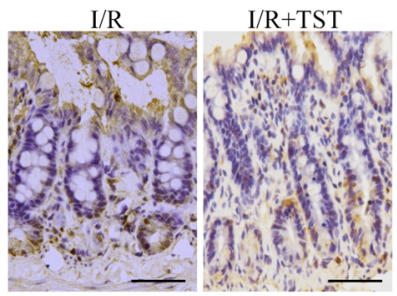

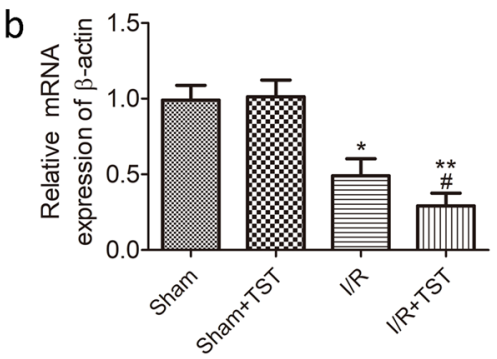

d
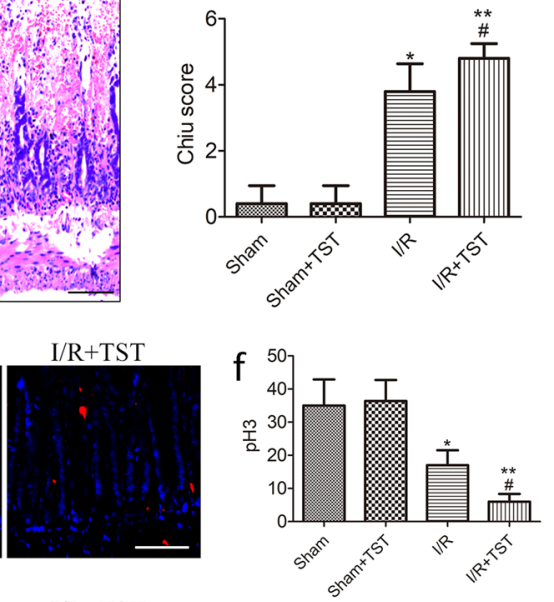

h

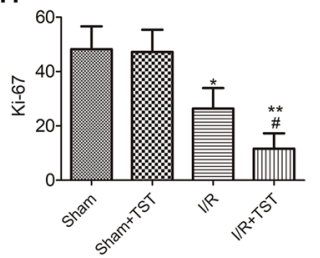

Fig. 2 Reduced expression of FoxM1 by TST inhibits intestinal epithelial proliferation after I/R in vivo. a Representative protein levels of FoxM1 in the intestinal tissues of different groups $(n=6)$. $\mathbf{b}$ Representative mRNA levels of FoxM1 in the intestinal tissues of different groups ( $n=6$ ). c, $\mathbf{d}$ HE staining and Chiu scores of intestinal tissues $(n=5)$ (bar $=50 \mu \mathrm{m})$. e, $\mathbf{f}$ Immunofluorescence staining for pH3 in the intestinal tissues of different groups $(n=5)$ (bar $=100 \mu \mathrm{m})$. $\mathbf{g}$, $\mathbf{h}$ Immunohistochemical staining for Ki-67 $(n=5)(b a r=100 \mu m)$. The values are presented as the means \pm SDs. ${ }^{* *} P<0.01$ compared to the sham group, ${ }^{\#} P<0.05$ compared to the $\mathrm{I} / \mathrm{R}$ group, ${ }^{\# \#} P<0.01$ compared to the I/R group

that FoxM1 bound to site 1 of the upstream region of Nurr1 TSS directly, but not site 2 (Fig. 7b). Next, we performed dual-luciferase reporter assays to determine whether FoxM1 binds site 1 of the Nurr1 promoter. IEC- 6 cells were cultured and transfected with plasmid containing wild-type or site 1 mutant Nurr1 promoter. Luciferase activity of cells expressing the wild-type Nurr1 promoter containing binding site 1 was activated by FoxM1 expression (Fig. 7c). These results revealed that the integrity of binding site 1 upstream of the Nurr1 TSS is required for FoxM1-mediated activation of Nurr1 expression.

\section{Downregulation of FoxM1/Nurr1 in the ischemic intestine of clinical patients}

To determine the function of FoxM1 in the proliferation of intestinal epithelial cells of the human ischemic intestine, we investigated proliferation in the human ischemic intestine by $\mathrm{HE}$ (Fig. 8a, b) and immunohistochemical (Fig. 8c, d) staining and the expression of FoxM1 and Nurr1 in clinical patients with intestinal ischemia (Fig. 8e, f). We observed the decreased expression of FoxM1 and Nurr1 and the expression of Ki-67 in the human ischemic intestine. These results were similar to those in the rat model of intestinal I/R injury. 
a
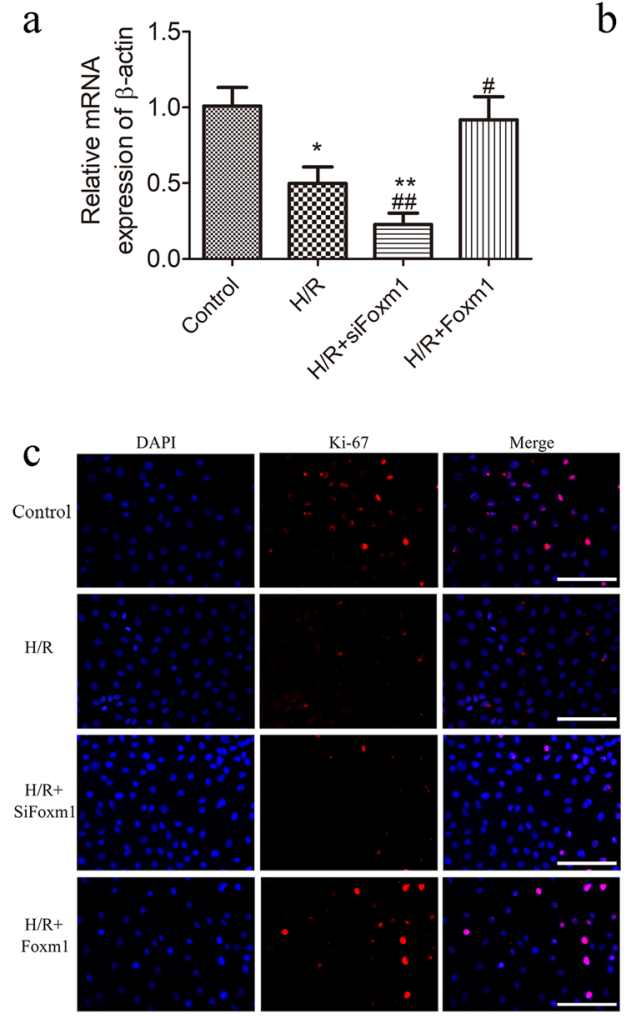

b
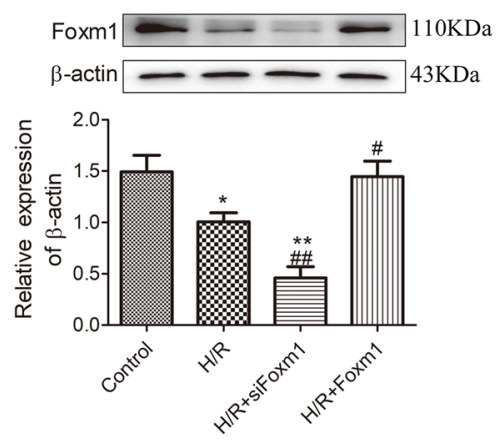

d

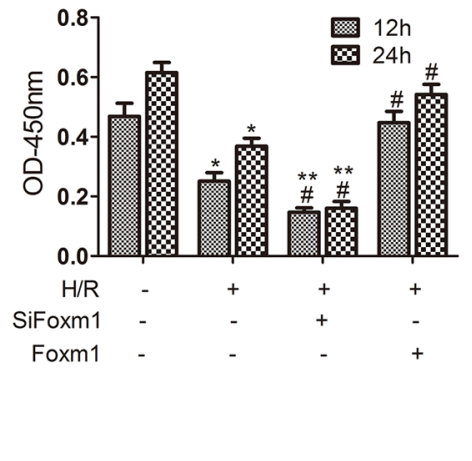

Fig. 3 FoxM1 promotes IEC-6 cell proliferation after H/R injury. a Representative mRNA levels of FoxM1 in IEC-6 cells in different groups ( $n=6$ ). b Representative protein levels of FoxM1 in IEC-6 cells in different groups $(n=6)$. c Immunofluorescence staining for pH3 in IEC-6 cells in different groups $(n=5)(\mathrm{bar}=100 \mu \mathrm{m})$. d The CCK-8 assay was used to examine the cell proliferation of IEC- 6 cells in different groups after hypoxia for $6 \mathrm{~h}$ and $12 \mathrm{~h}$ or $24 \mathrm{~h}$ of reoxygenation $(n=6)$. The values are presented as the means \pm SDs. ${ }^{*} P<0.05$ compared to the control group, ${ }^{* *} P<0.01$ compared to the control group, ${ }^{\#} P<0.05$ compared to the I/R group, ${ }^{\# \# P}<0.01$ compared to the I/R group

\section{Discussion}

Intestinal $\mathrm{I} / \mathrm{R}$ injury, a serious condition in intensive care units and among vascular surgery patients, is characterized by mucosal barrier damage. Once intestinal mucosal damage occurs, new intestinal crypt epithelial cells proliferate and migrate to the villus and subsequently restore proper mucosal barrier function ${ }^{26,27}$. Previous studies have shown that several genes are activated during the proliferation of intestinal epithelial cells after injury ${ }^{28,29}$. However, the intrinsic mechanism of intestinal epithelial proliferation remains unknown. Our previous study showed that Nurr1 is involved in intestinal regeneration after $\mathrm{I} / \mathrm{R}$ injury by directly inhibiting $\mathrm{p} 21$ expres$\operatorname{sion}^{23}$. Our study is the first to find that (1) FoxM1 can be induced during intestinal mucosa regeneration after $I / R$ injury, (2) FoxM1 promotes intestinal epithelial cell proliferation by promoting Nurr1 gene expression, and (3) Nurr1 is a novel downstream effector of FoxM1 that can be directly activated by FoxM1 and mediate the ability of FoxM1 to promote intestinal regeneration after I/R injury. Intestinal epithelial cells proliferate to rebuild the proper structure of the epithelium after I/R injury, and nuclear factors are involved in intestinal mucosa regeneration after injury ${ }^{30-32}$. FoxM1 is involved in the regeneration of several organs after injury ${ }^{33,34}$. As a nuclear factor, FoxM1 promotes organ regeneration after injury via its transcriptional regulation of downstream genes $^{35,36}$. FoxM1 is highly expressed in intestinal crypts, where it stimulates proliferation by promoting cell cycle entry into $S$ phase and $M$ phase, and may be a key target for regeneration after injury to some organs. In addition, inflammation can promote tissue regeneration after injury through poorly understood mechanisms ${ }^{37}$. As a transcription factor, FoxM1 acts as a critical mediator of the inflammatory response. Zeng et al. revealed that the inhibition of FoxM1 suppresses the production of inflammatory factors including tumor necrosis factor- $\alpha$ and IL-6 in osteoarthritis. The inflammatory mediators nitric oxide, prostaglandin E2 and cyclooxygenase-2 were also repressed by FoxM1 knockdown ${ }^{38}$. It has been reported that inflammatory diseases drive tissue hypoxia $^{39,40}$. FoxM1 is involved in tissue regeneration after injury ${ }^{33,34}$. In our study, FoxM1 was induced in intestinal regeneration after $I / R$ injury. In addition, the 

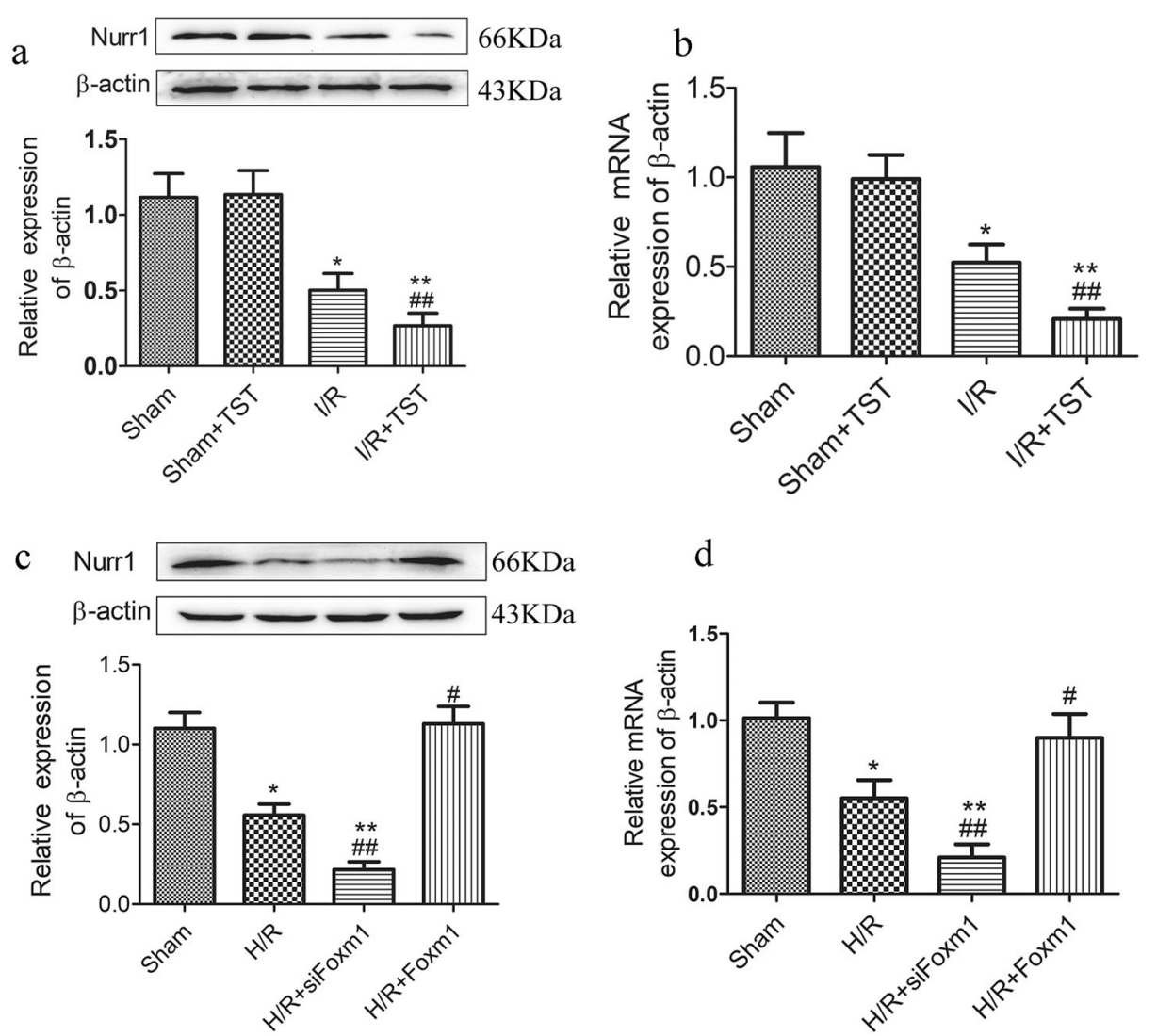

Fig. 4 Nurr1 is induced by FoxM1 intestinal regeneration after I/R in vivo and in vitro. a Representative protein levels of Nurr 1 in the rats of different groups determined by western blotting $(n=6)$. $\mathbf{b}$ Representative mRNA levels of Nurr 1 in the rats of different groups determined by realtime PCR $(n=6)$. c Representative protein levels of Nurr1 in IEC-6 cells of different groups determined by western blotting $(n=6)$. $\mathbf{d}$ Representative mRNA levels of Nurr1 in IEC- 6 cells in different groups determined by real-time PCR $(n=6)$. The values are presented as the means \pm SDs. ${ }^{*} P<0.05$ compared to the sham group, ${ }^{* *} P<0.01$ compared to the sham group, ${ }^{\#} P<0.05$ compared to the I/R group, ${ }^{\# \#} P<0.01$ compared to the I/R group

expression of FoxM1 was consistent with that of Ki-67 and $\mathrm{pH} 3$ in the intestinal mucosa after $\mathrm{I} / \mathrm{R}$ injury. These results revealed that the expression of FoxM1 is closely associated with intestinal epithelial cell proliferation. Furthermore, inhibition of the expression of FoxM1 resulted in the reduced proliferation of intestinal epithelial cells, and the activation of FoxM1 promoted increased cell proliferation. These data suggested that FoxM1 promotes intestinal mucosa regeneration after I/R injury.

Previous studies have shown that FoxM1 induces the expression of proliferation-associated genes that regulate cell cycle progression and promote cell proliferation in various tissues. Zhao et al. reported that overexpression of FoxM1 induces the expression of cell cycle-associated genes and promotes the proliferation of lung endothelial cells and epithelial cells in a different model of inflammatory lung injury ${ }^{41,42}$. Hou et al. reported that E2F1 target genes and other genes that promote $\mathrm{S}$ phase transition were increased when FoxM1 was absent in hematopoietic stem cells, providing molecular evidence of the promotion of $\mathrm{S}$ phase progression in hematopoietic stem cells and increased proliferation of hematopoietic stem cells. Moreover, downregulation of FoxM1 led to the loss of Nurr $1^{17}$. Interestingly, our previous studies showed that the loss of Nurr1 markedly impairs intestinal epithelial cell proliferation and is associated with the upregulation of $21^{18}$. In this study, we found that the ectopic expression of Nurr1 could reverse the inhibition of intestinal epithelial cell proliferation induced by FoxM1 deletion, indicating that these roles are likely mediated by Nurr1. Furthermore, for the first time, our results demonstrated the physiological significance of the overexpression of FoxM1 and resultant epithelial regeneration in the mechanism of intestinal epithelial cell repair following I/R injury. FoxM1 activates Nurr1 expression, and the forced expression of Nurr1 reverses the inhibitory effect of FoxM1 deficiency on the proliferation of intestinal epithelial cells. Taken together, these results suggest that FoxM1 regulates intestinal epithelial cell proliferation through activating Nurr1-mediated pathways.

Next, we examined the intrinsic molecular mechanism by which FoxM1 inhibits the expression of Nurr1. FoxM1 
a
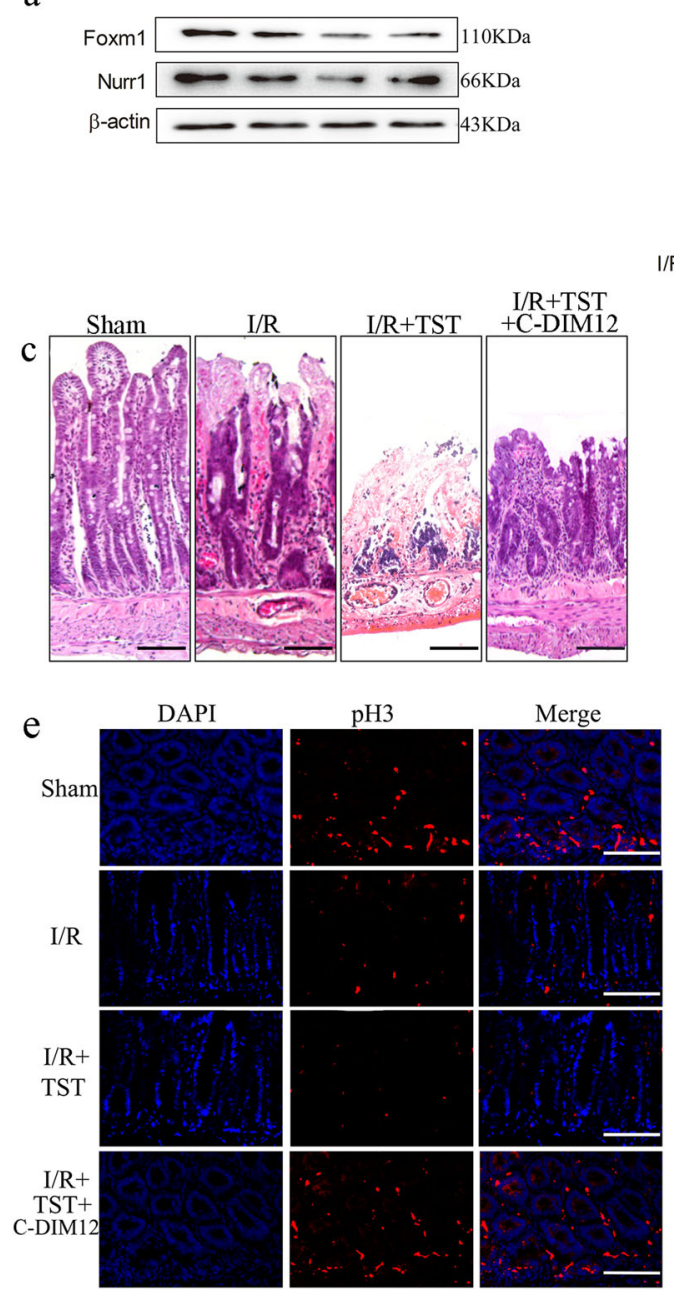

$\mathrm{b}$
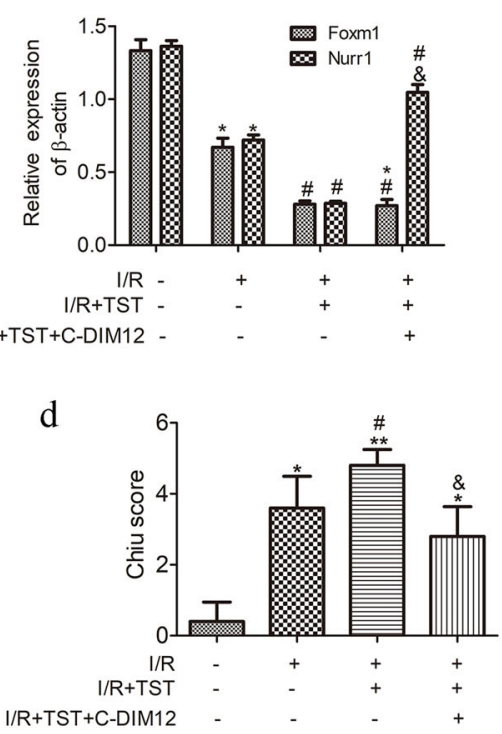

$\mathrm{f}$

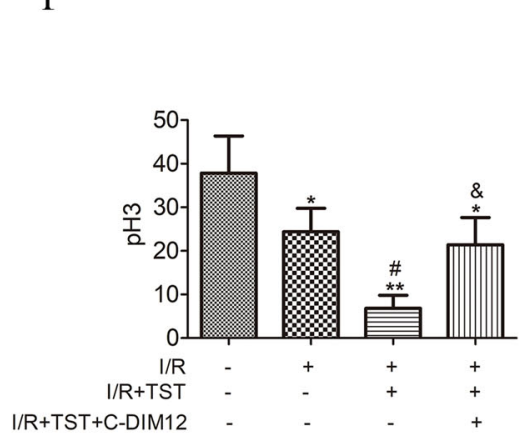

Fig. 5 Nurr1 mediates FoxM1 and promotes intestinal regeneration after I/R injury. $\mathbf{a}$, b Representative protein levels of FoxM1 in the intestinal tissues of different groups $(n=6)$. $\mathbf{c}, \mathbf{d}$ HE staining and Chiu scores of intestinal tissues $(n=5)(b a r=50 \mu \mathrm{m})$. e, $\mathbf{f}$ Immunofluorescence staining for $\mathrm{pH} 3$ in the intestinal tissues of different groups $(n=5)(\mathrm{bar}=50 \mu \mathrm{m})$. The values are presented as the means $\pm \mathrm{SDs}$. ${ }^{*} P<0.05$ compared to the sham group, ${ }^{*} P<0.01$ compared to the sham group, ${ }^{\#} P<0.05$ compared to the $\mathrm{I} / \mathrm{R}$ group, ${ }^{\&} P<0.05$ compared to the $\mathrm{I} / \mathrm{R}+\mathrm{TST}$ group

can bind to and transactivate target promoters by recognizing and binding to a specific FoxM1-binding site. Hou et al. identified two putative FoxM1-binding sites upstream of the Nurr1 TSS (-5585 to -5576 and -167 to $-159)$ and showed that consensus site $1(-5585$ to -5576$)$ in the promoter of Nurr1 was required for FoxM1mediated activation of Nurr1 expression (-5585 to -5576) in a mouse model ${ }^{17}$. Using bioinformatic analysis, we found a consensus FoxM1-binding site in the Nurr1 promoter that interacted with FoxM1. The results of subsequent ChIP and luciferase assays showed that the binding of FoxM1 to this site activated Nurr1 transcription. This evidence revealed the direct promotion of Nurr1 expression at the transcriptional level by FoxM1.
However, other transcriptional factors that involve additional regulatory mechanisms cannot be excluded.

The role of FoxM1 in intestinal I/R injury is unknown. By analyzing the expression of FoxM1 in clinical patients with superior mesenteric artery embolus, we found that FoxM1 was significantly downregulated in the ischemic mucosa of intestinal I/R patients. In our previous study, we tested Nurr1 expression was downregulated in the intestinal mucosa after ischemic injury ${ }^{18}$. More importantly, the expression of FoxM1 and Nurr1 was reduced along with the expression of $\mathrm{Ki}-67$ in the ischemic intestinal mucosa. Thus, our data implicated the critical role of FoxM1 in promoting human intestinal mucosa epithelial cells and suggested that the upregulation of 
a
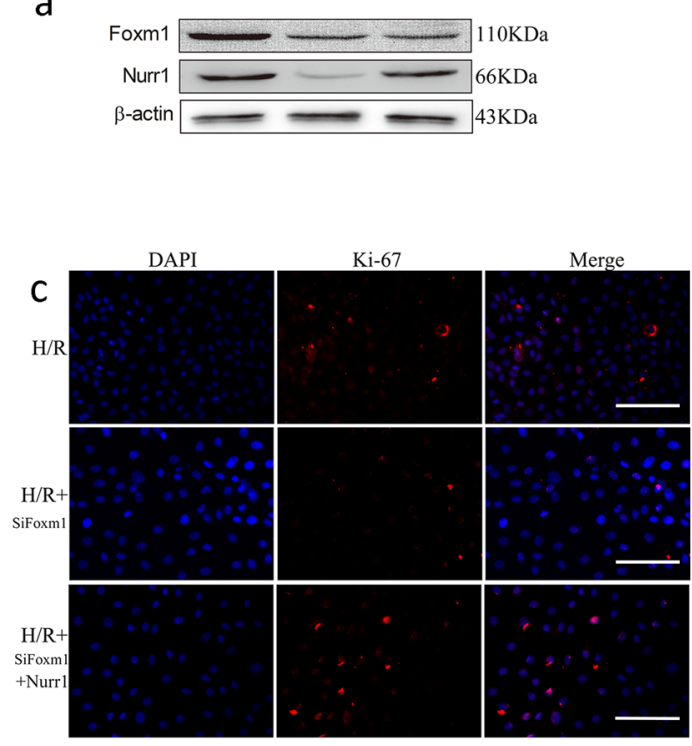

b

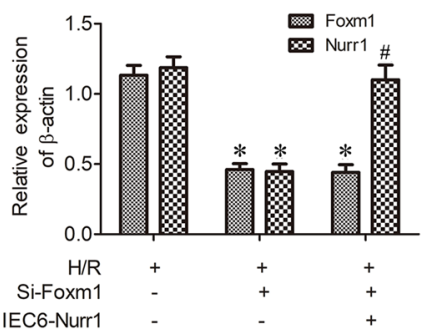

d

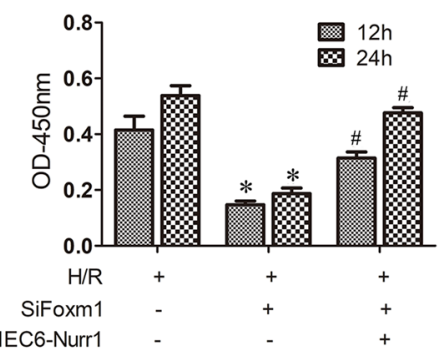

Fig. 6 FoxM1 promotes IEC- 6 cell proliferation after H/R injury in a Nurr1-dependent manner. $\mathbf{a}$, $\mathbf{b}$ Representative protein levels of FoxM1 in IEC-6 cells in different groups $(n=6)$. c Immunofluorescence staining for Ki-67 in IEC-6 cells in different groups $(n=5)($ bar $=100 \mu \mathrm{m})$. d The CCK-8 assay was used to examine the cell proliferation of IEC- 6 cells in different groups $(n=6)$. The values are presented as the means \pm SDs. ${ }^{*} P<0.05$ compared to the H/R group, ${ }^{\#} P<0.05$ compared to the $H / R+$ siFoxM1 group

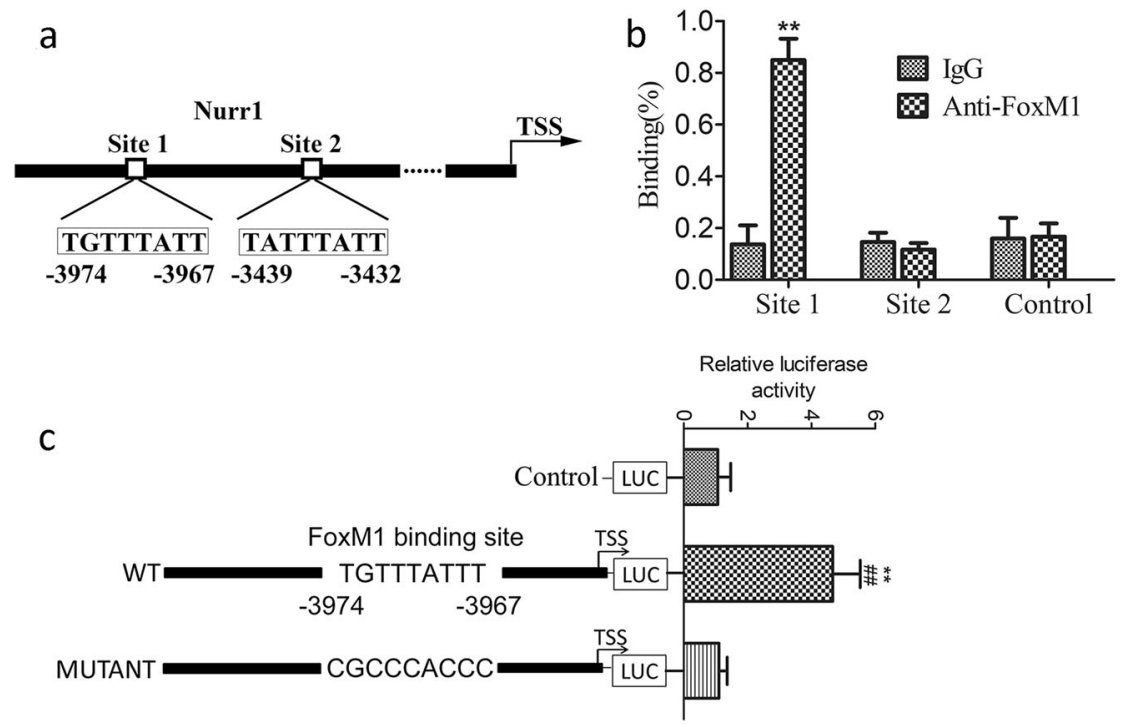

Fig. 7 FoxM1 regulates Nurr1 expression by directly inhibiting Nurr1 expression. a Schematic diagram of the wild-type (WT) rat Nurr1 upstream promoter region showing the predicted FoxM1-binding regions: site 1 and site 2 . TSS indicates the transcription start site. $\mathbf{b}$ Endogenous binding of FoxM1 to the upstream region of Nurr1 in IEC-6 cells after H/R injury as determined by ChIP assay. IgG was used as a negative control. c The TGTTATTT core motif was mutated into CGCCCACCC in the mutant Nurr1 promoter luciferase construct. Luciferase reporter assays: IEC-6 cells were transfected with wild-type or mutated Nurr1 promoter luciferase constructs and either FoxM1 or pEX-3 vectors

Nurr1 expression contributes to the proliferation of epithelial cells of the human intestinal mucosa.

In summary, we show that FoxM1 acts as a critical regulator of intestinal mucosa regeneration after $I / R$ injury by promoting the expression of Nurr1. Moreover, we revealed that FoxM1 promotes intestinal mucosa epithelial cell proliferation by directly binding the promoter of Nurr1 and promoting the transcription of Nurr1. 


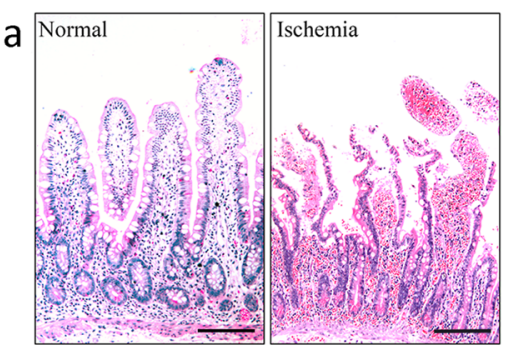

d

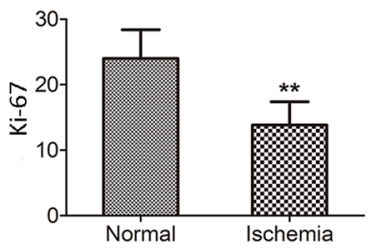

b

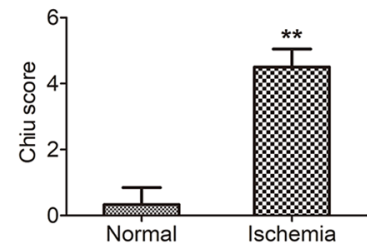

e

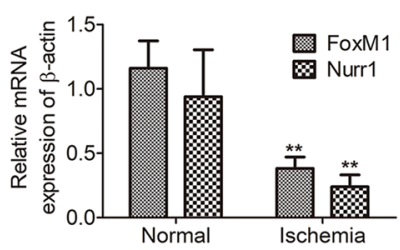

$c$

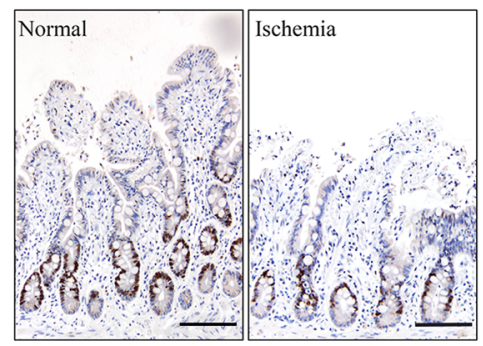

f
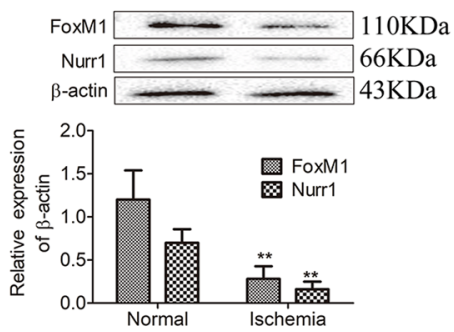

Fig. 8 Downregulation of FoxM1/Nurr1 in the ischemic intestine of clinical patients. $\mathbf{a}$, $\mathbf{b}$ Histology and Chiu scores of ischemic intestinal tissues from clinical patients ( $\mathrm{bar}=50 \mu \mathrm{m})$. c $\mathbf{d}$ Immunohistochemical staining for Ki-67 (bar $=50 \mu \mathrm{m})$. e Representative mRNA levels of FoxM1 and Nurr1 $(n=5)$. $\mathbf{f}$ Representative protein levels of FoxM1 and Nurr1 $(n=5)$. The values are presented as the means \pm SDs. ${ }^{*} P<0.01$ compared to normal intestinal tissue

Together, the results of our study reveal the important role of the FoxM1/Nurr1 signaling pathway in promoting intestinal regeneration after $\mathrm{I} / \mathrm{R}$ injury. Therefore, we believe that the FoxM1/Nurr1 signaling pathway is a novel therapeutic target for intestinal $I / R$ injury and related clinical diseases.

\section{Acknowledgements}

This work was supported by a grant from the National Natural Science Foundation of China (grant number 81700465).

\section{Author details}

'Department of Gastroenterology Surgery, The Dalian Municipal Central Hospital Affiliated of Dalian Medical University, 116033 Dalian, China. ${ }^{2}$ Institute of Integrative Medicine, Dalian Medical University, 116044 Dalian, China. ${ }^{3}$ Department of Neurology, The First Affiliated Hospital of Dalian Medical University, 116011 Dalian, China. ${ }^{4}$ Department of Neurosurgery, The Second Affiliated Hospital of Dalian Medical University, 116027 Dalian, China

\section{Author contributions}

G.Z. and J.G. designed this research. G.Z., J.G., T.Z. D.W. and B.L. performed the research. G.Z., J.G., T.Z. and N.C. analyzed the data. G.Z., J.G. and X.Z. participated in sample collection. G.Z. and J.G. wrote and revised the paper. G.Z. provided financial support. All authors reviewed and approved the paper.

\section{Conflict of interest}

The authors declare that they have no conflict of interest.

\section{Publisher's note}

Springer Nature remains neutral with regard to jurisdictional claims in published maps and institutional affiliations.

Supplementary information accompanies this paper at https://doi.org/ 10.1038/s12276-019-0343-y.
Received: 8 April 2019 Revised: 12 July 2019 Accepted: 7 August 2019.

Published online: 8 November 2019

\section{References}

1. Chassin, C. et al. MicroRNA-146a-mediated downregulation of IRAK1 protects mouse and human small intestine against ischemia/reperfusion injury. EMBO Mol. Med. 4, 1308-1319 (2012).

2. Kannan, L. et al. R-spondin3 prevents mesenteric ischemia/ reperfusioninduced tissue damage by tightening endothelium and preventing vascular leakage. Proc. Natl Acad. Sci. USA v110, 14348-14353 (2013).

3. Sun, L. X. et al. Effects of Ganoderma lucidum polysaccharides on IEC-6 cell proliferation, migration and morphology of differentiation benefiting intestinal epithelium healing in vitro. J. Pharm. Pharmacol. 63, 1595-1603 (2011).

4. Oldenburg, W. A. et al. Acute mesenteric ischemia: a clinical review. Arch. Intern. Med. 164, 1054-1062 (2004).

5. Berlanga, J. et al. Prophylactic use of epidermal growth factor reduces ischemia/reperfusion intestinal damage. Am. J. Pathol. 161, 373-379 (2002).

6. Brandt, L. J. et al. AGA technical review on intestinal ischemia. American Gastrointestinal Association. Gastroenterology 118, 954-968 (2000).

7. Waseem, T. et al. Ghrelin promotes intestinal epithelial cell proliferation through PI3K/Akt pathway and EGFR trans-activation both converging to ERK 1/2 phosphorylation. Peptides 52, 113-121 (2014).

8. $\mathrm{Ye}, \mathrm{H}$. et al. Hepatocyte nuclear factor $3 /$ fork head homolog 11 is expressed in proliferating epithelial and mesenchymal cells of embryonic and adult tissues. Mol. Cell Biol. 17, 1626-16241 (1997).

9. Kalin, T. V. et al. Forkhead Box $\mathrm{m} 1$ transcription factor is required for perinatal lung function. Proc. Natl Acad. Sci. USA 105, 19330-19335 (2008).

10. Ustiyan, V. et al. Forkhead box M1 transcriptional factor is required for smooth muscle cells during embryonic development of blood vessels and esophagus. Dev. Biol. 336, 266-279 (2009).

11. Kalin, T. V. et al. Multiple faces of FoxM1 transcription factor: lessons from transgenic mouse models. Cell Cycle 10, 396-405 (2011).

12. Costa, R. H. FoxM1 dances with mitosis. Nat. Cell Biol. 7, 108-110 (2005).

13. Laoukili, J. et al. FoxM1 is required for execution of the mitotic programme and chromosome stability. Nat. Cell Biol. 7, 126-136 (2005).

14. Wang, X. et al. The Forkhead Box m1b transcription factor is essential for hepatocyte DNA replication and mitosis during mouse liver regeneration. Proc. Natl Acad. Sci. USA 99, 16881-16886 (2002). 
15. Ackermann Misfeldt, A. et al. Beta-cell proliferation, but not neogenesis, following 60\% partial pancreatectomy is impaired in the absence of FoxM1. Diabetes 57, 3069-3077 (2008).

16. $\mathrm{Ye}, \mathrm{H}$. et al. Premature expression of the winged helix transcription factor $\mathrm{HFH}-$ $11 \mathrm{~B}$ in regenerating mouse liver accelerates hepatocyte entry into $\mathrm{S}$ phase. Mol. Cell Biol. 19, 8570-8580 (1999).

17. Hou, Y. et al. The transcription factor Foxm1 is essential for the quiescence and maintenance of hematopoietic stem cells. Nat. Immunol. 16, 810-818 (2015).

18. $\mathrm{Zu}, \mathrm{G}$. et al. Nurr1 promotes intestinal regeneration after ischemia/reperfusion injury by inhibiting the expression of p21 (Waf1/Cip1). J. Mol. Med. 95, 83-95 (2017).

19. $\mathrm{Zu}, \mathrm{G}$. et al. Protective effects of ginsenoside $\mathrm{Rg} 1 \mathrm{on}$ intestinal ischemia/ reperfusion injury-induced oxidative stress and apoptosis via activation of the Wnt/B-catenin pathway. Sci. Rep. 6, 38480 (2016).

20. Weiler, S. M. E. et al. Induction of chromosome instability by activation of yesassociated protein and forkhead box M1 in liver cancer. Gastroenterology $\mathbf{1 5 2}$, 2037-2051 (2017).

21. Dai, Z. et al. Endothelial and smooth muscle cell interaction via FoxM1 Slgnaling Mediates Vascular Remodeling and Pulmonary Hypertension. Am. J. Respir. Crit. Care Med. 198, 788-802 (2018).

22. De Miranda, B. R. et al. Neuroprotective efficacy and pharmacokinetic behavior of novel anti-inflammatory para-phenyl substituted diindolylmethanes in a mouse model of Parkinson's disease. J. Pharm. Exp. Ther. 345, 125-138 (2013).

23. Liu, L. et al. miR-381-3p knockdown improves intestinal epithelial proliferation and barrier function after intestinal ischemia/ reperfusion injury by targeting nurr1. Cell Death Dis. 9, 411 (2018).

24. Yang, N. et al. FOXM1 recruits nuclear Aurora kinase $A$ to participate in a positive feedback loop essential for the self-renewal of breast cancer stem cells. Oncogene 36, 3428-3440 (2017).

25. Wierstra, l. et al. Despite its strong transactivation domain, transcription factor FOXM1c is kept almost inactive by two different inhibitory domains. Biol. Chem. 387, 963-976 (2006).

26. Rijke, R. P. et al. The effect of ischemic villus cell damage on crypt cell proliferation in the small intestine: evidence for a feedback control mechanism. Gastroenterology 71, 786-792 (1976).

27. Yeh, K. Y. et al. Expression of intestinal brush-border membrane hydrolases and ferritin after segmental ischemia-reperfusion in rats. Am. J. Physiol. 275, G572-G583 (1998)

28. Shima, Y. et al. Increased expression of c-fos and c-jun in the rat small intestinal epithelium after ischemia-reperfusion injury: a possible correlation with the proliferation or apoptosis of intestinal epithelial cells. J. Pediatr. Surg. 41, 830-836 (2006).
29. Itoh, $\mathrm{H}$. et al. Regeneration of small intestinal mucosa after acute ischemiareperfusion injury. Diq. Dis. Sci. 47, 2704-2710 (2002).

30. El-Assal, O. N. et al. HB-EGF enhances restitution after intestinal ischemia/ reperfusion via PI3K/Akt and MEK/ERK1/2 activation. Gastroenterology 129 609-625 (2005).

31. Ren, F. et al. Drosophila Myc integrates multiple signaling pathways to regulate intestinal stem cell proliferation during midgut regeneration. Cell Res. 23, 1133-1146 (2013)

32. Macho-Fernandez, E. et al. Lymphotoxin beta receptor signaling limits mucosal damage through driving IL-23 production by epithelial cells. Mucosal Immunol. 8, 403-413 (2015).

33. Izumi, T. et al. Vagus-macrophage-hepatocyte link promotes post-injury liver regeneration and whole-body survival through hepatic FoxM1 activation. Nat. Commun. 9, 5300 (2018).

34. Cheng, Y. Y. et al. Reprogramming-derived gene cocktail increases cardiomyocyte proliferation for heart regeneration. EMBO Mol. Med. 9, 251-264 (2017).

35. Sato, Y. et al. Regulatory signatures of liver regeneration distilled by integrative analysis of mRNA, histone methylation, and proteomics. J. Biol. Chem. 292, 8019-8037 (2017)

36. Krupczak-Hollis, K. et al. The mouse Forkhead Box $\mathrm{m} 1$ transcription factor is essential for hepatoblast mitosis and development of intrahepatic bile ducts and vessels during liver morphogenesis. Dev. Biol. 276, 74-88 (2004).

37. Taniguchi, $\mathrm{K}$. et al. A gp130-Src-YAP module links inflammation to epithelial regeneration. Nature 519, 57-62 (2015).

38. Zeng, R. M. et al. Knockdown of FOXM1 attenuates inflammatory response in human osteoarthritis chondrocytes. Int. Immunopharmacol. 68, 74-80 (2019).

39. Qi, L. et al. [Hypoxia/reoxygenation and lipopolysaccharide induced nuclear factor-KB and hypoxia-inducible factor-1a signaling pathways in intestinal epithelial cell injury and the interventional effect of emodin]. Zhonghua Wei Zhong Bing. Ji Jiu Yi Xue. 26, 409-414 (2014).

40. Tian, S. et al. HDAC inhibitor valproic acid protects heart function through Foxm1 pathway after acute myocardial infarction. EBioMedicine 39, 83-94 (2019).

41. Kalinichenko, V. V. et al. Ubiquitous expression of the forkhead box M1B transgene accelerates proliferation of distinct pulmonary cell types following lung injury. J. Biol. Chem. 278, 37888-37894 (2003).

42. Zhao, Y. D. et al. Endothelial FoxM1 mediates bone marrow progenitor cellinduced vascular repair and resolution of inflammation following inflammatory lung injury. Stem Cells 32, 1855-1864 (2014). 